\title{
Environmental Effects on the Faint End of the Luminosity Function
}

\author{
S. Phillipps, J.B. Jones
}

Department of Physics, University of Bristol, Bristol, UK

R.M. Smith

Department of Physics and Astronomy, University of Wales, Cardiff, $U K$

W.J. Couch, S.P. Driver

School of Physics, University of New South Wales, Sydney, Australia

\begin{abstract}
Recent studies have demonstrated that many galaxy clusters have luminosity functions (LFs) which are steep at the faint end. However, it is equally clear that not all clusters have identical LFs. In this paper we explore whether the variation in LF shape correlates with other cluster or environmental properties.
\end{abstract}

\section{Introduction}

Much recent work has been devoted to measuring the galaxy luminosity function (LF) within rich clusters, particularly with regard to the faint end which has become accessible to detailed study through various technical and observational improvements (see the paper by Smith et al. in these proceedings). These studies suggest that the LF becomes steep (Schechter (1976) slope $\alpha \leq-1.5$ ) in many clusters, faintwards of about $M_{B}=-17.5$ or $M_{R} \simeq-19$ (for $H_{0}=$ $50 \mathrm{~km} \mathrm{~s}^{-1} \mathrm{Mpc}^{-1}$ ), where (generally low surface brightness) dwarfs begin to dominate (e.g. Smith, Driver \& Phillipps 1997; Trentham 1997a,b). Using deep CCD imaging from the Anglo-Australian Telescope, we have now extended this work (see Driver, Couch \& Phillipps 1998), in order to examine the luminosity distribution in and across a variety of Abell and ACO clusters. In particular, we were interested in any possible dependence of the dwarf population (specifically the ratio of the number of dwarfs to the number of giants) on cluster type or on position within the cluster.

\section{Dwarfs in Rich Clusters}

A number of papers (e.g. Driver et al. 1994; Smith et al. 1997; Wilson et al. 1997) have demonstrated remarkably similar dwarf populations in a number of morphologically similar, dense rich clusters like (and including) Coma. This similarity appears not only in the faint end slope of the LF, around $\alpha=-1.8$, 
but also in the point at which the steep slope cuts in, $M_{R} \simeq-19$ (i.e. about $\left.M^{*}+3.5\right)$. The latter implies equal ratios of dwarf to giant galaxy numbers in the different clusters.

However, there clearly do exist differences between some clusters. For example, several of the clusters in the Driver et al. (1998) sample do not show a conspicuous turn up at the faint end (see also Lopez-Cruz et al. 1997 for further examples). Either these clusters contain completely different types of dwarf galaxy population or, as we suggest, the turn up occurs at fainter magnitudes. For a composite giant plus dwarf LF, this is equivalent to a smaller number of dwarfs relative to giants.

To simplify the discussion, we will define the dwarf to giant ratio DGR as the number of galaxies with $-16.5 \geq M_{R} \geq-19.5$ compared to those with $\left.-19.5 \geq M_{R}\right)$,

i.e. $\quad \vec{D} G R=\frac{N\left(-16.5>M_{R}>-19.5\right)}{N\left(-19.5>M_{R}>-23.5\right)}$.

The DGR does not have any obvious dependence on cluster richness (Driver et al. 1998; see also Turner et al. 1993), but we can also check for variations with morphological characteristics of the clusters. For giant galaxies, it is well known that a cluster's structural and population characteristics are well correlated. For example, dense regular clusters are of early Bautz-Morgan type (dominated by cD galaxies) and have the highest fractions of giant ellipticals (Dressler 1980). In a similar way, we find that the DGR (i.e. the fraction of dwarfs) is smallest in these early Bautz-Morgan type clusters (Driver et al. 1998). Next consider the galaxy density. We can characterise the clusters by their central (giant) galaxy number densities, for instance the number of galaxies brighter than $M_{R}=-19.5$ within the central $1 \mathrm{Mpc}^{2}$ area. An alternative would be to use Dressler's (1980) measure of the average number of near neighbours. We then find (solid squares in Figure 1) that the clusters with the least prominent dwarf populations (low DGRs $\sim 1$ ) are just those with the highest projected galaxy densities (e.g. the Bautz-Morgan Type I-II cluster A3888). Previously, Turner et al. (1993) had noted that the rich but low density cluster A3574, which is very spiral rich (Willmer et al. 1991), had a very high ratio of low surface brightness (LSB) dwarfs to giants. This is now backed up by the observations of clusters like A204 which are dwarf rich (DGR 3), have low central densities and late B-M types (A204 is B-M III).

To extend the range of environments studied, we can add in further LF results from the literature (Figure 2). A problem here, of course, is the lack of homogeneity due to different observed wavebands, different object detection techniques and so forth. Nevertheless, we can explore the general trends. Several points are shown for surveys of Coma (hexagons). These surveys (Thompson \& Gregory 1993, Lobo et al. 1997, Secker \& Harris 1996 and Trentham 1998) cover different areas and hence different mean projected densities (see also the next section). All these lie close to the relation defined by our original data, with the larger area surveys having higher DGRs. Points (filled triangles) representing the rich B-M type I X-ray selected clusters studied by Lopez-Cruz et al. (1997) fall at somewhat lower DGR than most of our clusters at similar densities. However we should note that these clusters were selected (from a larger unpublished sample) only if they had LFs well fitted by a single Schechter function. This obviously precludes clusters with steep LF turn-ups at intermediate magnitudes 


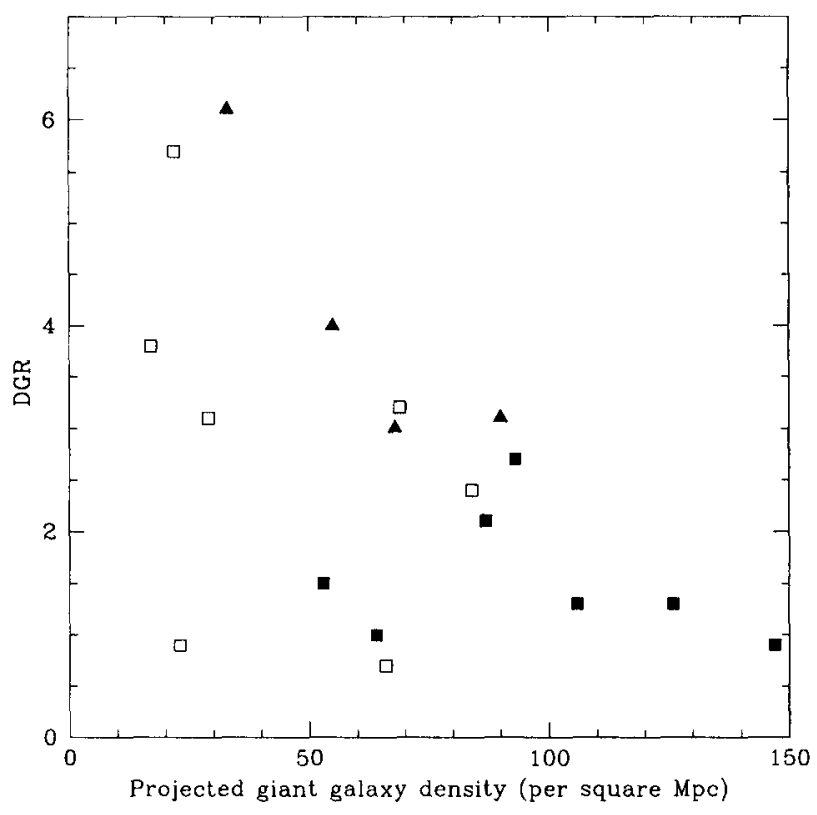

Figure 1. Variation of the dwarf-to-giant ratio (DGR), as defined in the text, with projected density of cluster giants (per square $\mathrm{Mpc}$ ). Solid boxes represent the central 1 square Mpc regions of the clusters, the open boxes the outer regions (data from Driver at al 1998). The triangles show the variation over a wider range of radii for Abell 2554 (data from Smith et al 1997). Note that typical error bars (due to the combination of Poisson errors and background subtraction errors) are $10 \%$ in density and $20 \%$ in DGR for the denser regions, rising to $30 \%$ in density and $50 \%$ in DGR at the lowest densities (and hence object numbers). The outlier at low density and low DGR (the outskirts of A22) has a very large error in DGR $(\sim 100 \%)$. 
and hence rules out high DGRs. The one comparison cluster they do show with a turn up (A1569 at DGR $\simeq 4.2$ ) clearly supports our overall trend.

Ferguson \& Sandage $(1991=$ FS), on the other hand, deduced a trend in the opposite direction, from a study of fairly poor groups and clusters, with the early type dwarf-to-giant ratio increasing for denser clusters. However, this is not necessarily as contradictory to the present result as it might initially appear. For instance, FS select their dwarfs morphologically, not by luminosity (morphologically classified dwarfs and giants significantly overlap in luminosity) and they also concentrate solely on early type dwarfs. If, as we might expect, low density regions have significant numbers of late type dwarf irregulars (e.g. Thuan et al. 1991), then the FS definition of DGR may give a lower value than ours for these regions. Furthermore FS calculate their projected densities from all detected galaxies, down to very faint dwarfs. Regions with high DGR will therefore be forced to much higher densities than we would calculate for giants only. These two effects may go much of the way to reconciling our respective results. This is illustrated by the open triangles in Figure 2, which are an attempt to place the FS points on our system; magnitudes have been adjusted approximately for the different wavebands, DGRs have been estimated from the LFs and the cluster central densities (from Ferguson \& Sandage 1990) have been scaled down by the fraction of their overall galaxy counts which are giants (by our luminosity definition). Given the uncertainties in the translation, most of the FS points then lie close to those of our overall distribution. Finally, a field LF with a steep faint end tail $(\alpha \simeq-1.5$; e.g. Marzke, Huchra \& Geller 1994, Zucca et al. 1997, Morgan, Smith \& Phillipps 1998)) would also give a point (filled pentagon) at $\mathrm{DGR} \simeq 4$, again consistent with the trend seen in the clusters.

Nevertheless, there are exceptions. The FS points of lowest density (the Leo and Dorado groups) also have low DGR (and lie close to our main 'outlier', the point for the outer region of A22). The Local Group (shown by the star) would also be in this regime, at low density and DGR $=2$, as would the 'conventional' field with $\alpha \simeq-1.1$ (Efstathiou, Ellis \& Peterson 1988; Loveday et al. 1992) and hence $\mathrm{DGR} \simeq 1.5$ (open pentagon). This may suggest that at very low density the trend is reversed (i.e. is in the direction seen by FS), or that the cosmic (and/or statistical) scatter becomes large. More data in the very low density regime is probably required before we can make a definitive statement on a possible reversal of the slope of the DGR versus density relation. In particular, the scatter in the derived faint end of the field LF between different surveys (see, e.g., the recent discussion in Metcalfe et al. 1998) precludes using this to tie down the low density end of the plot.

\subsection{Population Gradients}

It was suggested by the results on A2554 (Smith et al. 1997), that the dwarf population was more spatially extended than that of the giants, i.e. the dwarf to giant ratio increased outwards. This type of population gradient has now been confirmed by the results in Driver et al. (1998) illustrated in Figure 1, where we contrast the inner $1 \mathrm{Mpc}^{2}$ areas (solid symbols) with the outer regions of the same clusters (open symbols). The triangles show in slightly more detail the run of DGR with radius (and hence density) across A2554. A similar effect can be seen for Coma in Figure 2 and can explain the discrepancy between the 


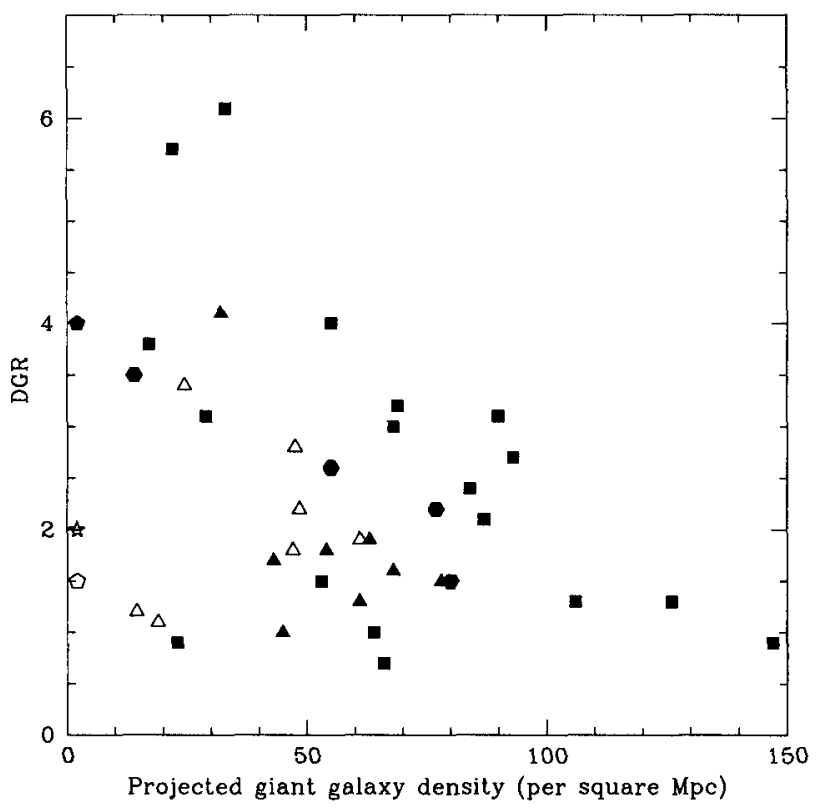

Figure 2. As Figure 1, but including data from other observers. Squares are our data repeated from Figure 1, hexagons are for various Coma surveys detailed in the text, filled triangles are from Lopez-Cruz's sample and open triangles are for Ferguson and Sandage's poor clusters and groups. The open pentagon at low density represents a conventional 'flat' field LF, the filled pentagon a possible steep $(\alpha \simeq-1.5)$ field LF and the Local Group is represented by the star at DGR $=2$. 
LFs derived for the core and as against larger areas. It is found, too, in Virgo (Phillipps et al. 1998a; Jones et al., these proceedings), where the dwarf LSBG population has almost constant number density across the central areas while the giant density drops by a factor $\sim 3$.

\section{A Dwarf Population Density Relation}

The obvious synthesis of the above results is a relationship between the local galaxy density and the fraction of dwarfs (i.e. the relative amplitude of the dwarf LF). The inner, densest parts of rich clusters have the smallest fraction of dwarfs, while loose clusters and the outer parts of regular clusters, where the density is low, have high dwarf fractions. It is particularly interesting to note the clear overlap region in Figure 1, where regions of low density on the outskirts of dense clusters (open squares) have similar DGRs to the regions of the same density at the centres of looser clusters (solid squares).

The proposed relation of course mimics the well known morphology - density relation (Dressler 1980), wherein the central parts of rich clusters have the highest early type galaxy fraction, this fraction then declining with decreasing local galaxy density. Putting the two relations together, it would also imply that dwarfs preferentially occur in the same environments as spirals. This would be in agreement with the weaker clustering of low luminosity systems in general (e.g. Loveday et al. 1995), as well as for spirals compared to ellipticals (Geller \& Davies 1976). Thuan et al. (1991) have previously discussed the similar spatial distributions of dwarfs (in particular dwarf irregulars) and larger late type systems.

\section{The Origin of the Relation}

As with the corresponding morphology - density relation for giant galaxies, the cause of our population - density relation could be either 'nature' or 'nurture', i.e. initial conditions or evolution. Some clues may be provided by the most recent semi-analytic models of galaxy formation, which have been able to account successfully for the excess of (giant) early type galaxies in dense environments (e.g. Baugh, Cole \& Frenk 1996), basically through different merging histories for different types of galaxy. Does this also work for the dwarfs?

The steep faint end slope of the LF appears to be a generic result of hierarchical clustering models (e.g. White \& Frenk 1991; Frenk et al. 1996; Kauffmann, Nusser \& Steinmetz $1997=$ KNS), so is naturally accounted for in the current generation of models. The general hierarchical formation picture envisages (mainly baryonic) galaxies forming at the cores of dark matter halos. The halos themselves merge according to the general Press-Schechter (1974) prescription to generate the present day halo mass function. However the galaxies can retain their individual identities within the growing dark halos, because of their much longer merging time scales. The accretion of small halos by a large one then results in the main galaxy (or cluster of galaxies, for very large mass halos) acquiring a number of smaller satellites (or the cluster gaining additional, less tightly bound, members). 
KNS have presented a detailed study of the distribution of the luminosities of galaxies expected to be associated with a single halo of given mass. We can thus easily compare the theoretically expected numbers of dwarf galaxies per unit giant galaxy luminosity with our empirical results (Phillipps et al. 1998b).

The KNS models mimic a "Milky Way system" (halo mass $5 \times 10^{12} M_{\odot}$ ), a sizeable group (halo mass $\left.5 \times 10^{13} M_{\odot}\right)$ and a cluster mass halo $\left(10^{15} M_{\odot}\right)$. Their results imply that the Milky Way and small group halos have similar numbers of dwarf galaxies per unit giant galaxy light, whereas the dense cluster environment has a much smaller number of dwarfs for a given total giant galaxy luminosity. Thus the predictions of the hierarchical models (which depend, of course, on the merger history of the galaxies) are in qualitative agreement with our empirical results if we identify loose clusters and the outskirts of rich clusters with a population of (infalling?) groups (cf. Abraham et al. 1996), whereas the central dense regions of the clusters originate from already massive dark halos. If we renormalise from unit galaxy light to an effective giant galaxy LF amplitude (see Phillipps et al. 1998b) then the actual expected ratios ( $\sim 1$ to a few) are also consistent with our observational results.

By inputting realistic star formation laws etc., KNS could further identify the galaxies in the most massive halos with old elliptical galaxies, and those in low mass halos with galaxies with continued star formation. This would imply the likelihood that our dwarfs in low density regions may still be star forming, or at least have had star formation in the relatively recent past (cf. Phillipps \& Driver 1995 and references therein). Note, too, that these galaxy formation models would also indicate that the usual (giant) morphology - density relation and our (dwarf) population - density relation do arise in basically the same way. Finally, we can see that if these semi-analytic models are reasonably believable, then we need not necessarily expect the field to be even richer in dwarfs than loose clusters; the dwarf to giant ratio seems to level off at the densities reached in fairly large groups.

\section{Summary}

To summarise, then, we suggest that the current data on the relative numbers of dwarf galaxies in different clusters and groups can be understood in terms of a general dwarf population versus local galaxy density relation, similar to the well known morphology - density relation for giants. Low density environments are the preferred habitat of low luminosity galaxies; in dense regions they occur in similar numbers to giants, but at low densities dwarfs dominate numerically by a large factor. This fits in with the general idea that low luminosity galaxies are less clustered than high luminosity ones (particularly giant ellipticals). Plausible theoretical justifications for the population - density relation can be found within the context of current semi-analytic models of hierarchical structure formation.

\section{References}

Abraham R.G., et al., 1996, ApJS, 471, 694

Baugh C.M., Cole S., Frenk C.S., 1996, MNRAS, 283, 1361 
Dressler A., 1980, ApJ, 236, 351

Driver S.P., Phillipps S., Davies J.I., Morgan I., Disney M.J., 1994, MNRAS, 268,393

Driver S.P., Couch W.J., Phillipps S., 1998, MNRAS, in press

Efstathiou G., Ellis R.S., Peterson B.A., 1988, MNRAS, 232, 431

Ferguson H.C., Sandage A., 1990, AJ, 100, 1

Ferguson H.C., Sandage A., 1991, AJ, 96, 1520

Frenk C.S., Evrard A.E., White S.D.M., Summers F.J., 1996, ApJ, 472, 460

Geller M.J., Davis M., 1976, ApJ, 208, 13

Jones J.B., Phillipps S., Schwartzenberg J.M., Parker Q.A., 1998, The Low Surface Brightness Universe, p.xxx

Kauffmann G., Nusser A., Steinmetz M., 1997, MNRAS, 286, 795

Lobo C., et al., 1997, A\&A, 317, 385

Lopez-Cruz O., Yee H.K.C., Brown J.P., Jones C., Forman W., 1997, ApJL, 475, L97

Loveday J., Maddox S.J., Efstathiou G., Peterson B.A., 1995, ApJ, 442, 457

Loveday J., Peterson B.A., Efstathiou G., Maddox S.J., 1992, ApJ, 390, 338

Marzke R., Huchra J.P., Geller M.J., 1994, ApJ, 428, 43

Metcalfe N., Ratcliffe A., Shanks T., Fong R., 1998, MNRAS, 294, 147

Morgan I., Smith R.M., Phillipps S., 1998, MNRAS, 295, 99

Phillipps S., Driver S.P., 1995, MNRAS, 274, 832

Phillipps S., Driver S.P., Couch W.J., Smith R.M., 1998b, ApJ, 498, L119

Phillipps S., Parker Q.A., Schwartzenberg J.M., Jones J.B., 1998a, ApJ, 493, L59

Press W.H., Schechter P.L., 1974, ApJ, 187, 425

Schechter P., 1976, ApJ, 203, 297

Secker J., Harris W.E., 1996, ApJ, 469, 623

Smith R.M., Driver S.P., Phillipps S., 1997, MNRAS, 287, 415

Smith R.M., Phillipps S., Driver S.P., Couch R.M., 1998, The Low Surface Brightness Universe, p.xxx

Thompson L.A., Gregory S.A., 1993, AJ, 106, 2197

Thuan T.X., Alimi J.M., Gott J.R., Schneider S.E., 1991, ApJ, 370, 25

Trentham N., 1997a, MNRAS, 286, 133

Trentham N., 1997b, MNRAS, 290, 334

Trentham N., 1998, MNRAS, 293, 71

Turner J.A., Phillipps S., Davies J.I., Disney M.J., 1993, MNRAS, 261, 39

White S.D.M., Frenk C.S., 1991, ApJ, 379, 52

Willmer C., Focardi P., Chan R., Pellegrini P., da Costa L., 1991, AJ, 101, 57

Wilson G, Smail I., Ellis R.S., Couch W.J., 1997, MNRAS, 284, 915

Zucca E., et al., 1997, in Wide-Field Spectroscopy, eds. Kontizas E. et al., Dordrecht; Reidel, p.247 